\title{
Coupled-Waves Approach to the Design and Analysis of Bragg and Photonic Crystal Annular Resonators
}

\author{
Jacob Scheuer, Member, IEEE, and Amnon Yariv, Life Fellow, IEEE
}

\begin{abstract}
We utilize a novel coupled-waves approach to design and analyze various types of annular resonators based on Bragg and photonic crystal reflectors. Optimal grating profiles and their corresponding modal fields are found for specific cases such as disk and defect mode (ring) resonators. An excellent match is found between our analytic approximations and exact results obtained by the transfer matrix formalism and finite-difference time-domain simulations.
\end{abstract}

Index Terms-Bragg resonators, integrated optics, perturbation methods, photonic crystals.

\section{INTRODUCTION}

$\mathbf{T}$ HE last few years have witnessed a substantial increase in research dealing with utilization of ring resonators for optical communication devices. Various ring and disk resonatorbased applications such as modulators [1], channel drop filters [2], and dispersion compensators [3] have been suggested and demonstrated.

Two important characteristics of the modes of ring resonators are the free spectral range (FSR) and the loss per revolution or, equivalently, the $Q$-factor. Our method for realizing high $Q$ is to attain tight confinement by utilizing Bragg reflection instead of total internal reflection (as in "conventional" resonators"). In this paper, we present a novel coupled-waves approach and employ it to design and analyze various types of annular resonators based on distributed feedback (DFB) reflectors. Disk and recently ring (defect) resonators based on Bragg reflection were analyzed in the past, both for laser and passive resonator applications [4]-[16], employing various techniques such as conformal mapping, coupled-wave approach, and field transfer matrices. Recently, a hexagonal waveguide ring resonator based on photonic crystal (PC) confinement on both sides of the waveguide was demonstrated experimentally [14]. However, this structure exploited the specific symmetry of the triangular lattice which enables low-loss $60^{\circ}$ abrupt turns in order to realize a closed resonator.

Circular symmetric structures, such as those depicted in Fig. 1, can be modeled accurately utilizing a transfer-matrix formalism [9]. However, noncircular symmetric structures, discussed in Section IV (see also Fig. 5), are difficult to analyze by transfer matrices. The analysis of such structures requires different tools, either numerical [such as finite-differ-

Manuscript received July 21, 2003; revised Augest 28, 2003. This work was supported by the U.S. Office of Naval Research and the U.S. Air Force Office of Scientific Research.

The authors are with the Department of Applied Physics, California Institute of Technology, Pasadena, CA 91125 USA (e-mail: koby@caltech.edu; ayariv@caltech.edu).

Digital Object Identifier 10.1109/JQE.2003.819548 ence time-domain (FDTD)] or the development of analytical approximated approaches. In addition, a simple analytical approach could shed light even on problems that can be analyzed accurately employing numerical techniques.

The basic geometry is illustrated in Fig. 1. A disk [Fig. 1(a)] or a circumferentially guiding defect [Fig. 1(b)] is located within a medium which consists of annular Bragg layers. Due to the circular geometry, the thicknesses of the layers, unlike in rectangular geometry, are not constant [18] and our task is to determine the thicknesses which lead to maximum confinement of the field in the disk or in the defect.

In Section II, we derive a coupled-waves formalism for designing such resonators and we attain a closed-form expression for the modal field. In Section III, we utilize this formalism to design and analyze Bragg reflector based disk and ring resonators. In Section IV, we design and analyze photonic crystal annular resonators and in Section $\mathrm{V}$ we discuss the results and summarize.

\section{COUPLED-WAVES EQuATIONS}

We consider a wave which propagates azimuthally in a twodimensional (2-D) structure as illustrated in Fig. 1: a ring/disk of dielectric material which is surrounded by radial perturbations of the dielectric coefficient $\varepsilon(\rho)$. All the electromagnetic field components can be expressed by the $z$ component of the electric and magnetic fields [17] which satisfy the scalar Helmholtz equation

$$
\left[\frac{1}{\rho} \frac{\partial}{\partial \rho}\left(\rho \frac{\partial}{\partial \rho}\right)+\frac{1}{\rho^{2}} \frac{\partial^{2}}{\partial \theta^{2}}+k_{0}^{2} n^{2}(\rho)\right]\left(\begin{array}{c}
E_{z} \\
H_{z}
\end{array}\right)=0
$$

where $\rho, z$, and $\theta$ are the radial, axial, and azimuthal coordinates, respectively, and $k_{0}$ is the wavenumber in vacuum.

In 2-D geometry, the solutions of (1) can be separated into two independent polarizations-TE, which includes $E_{z}, H_{\rho}$, and $H_{\theta}$, and TM comprising $H_{z}, E_{\rho}$, and $E_{\theta}$ [17]. In this paper, we focus on the TE polarization although similar analysis could be applied to the TM polarization. Although in many publications this polarization component is referred to as TM, we designate it as TE because it is perpendicular to the direction of the propagation $\theta$. We look for a modal solution of (1) which is resonant in the structure depicted in Fig. 1, with the following functional form:

$$
E_{z}=E(\rho) \cdot \exp (i m \theta)
$$

where $m$ is an integer. Substituting (2) into (1) leads to

$$
\rho^{2} \frac{d^{2} E}{d \rho^{2}}+\rho \frac{d E}{d \rho}+\left[k^{2}(\rho) \rho^{2}-m^{2}\right] E=0
$$

where $k(\rho)=k_{0} n(\rho)$. 


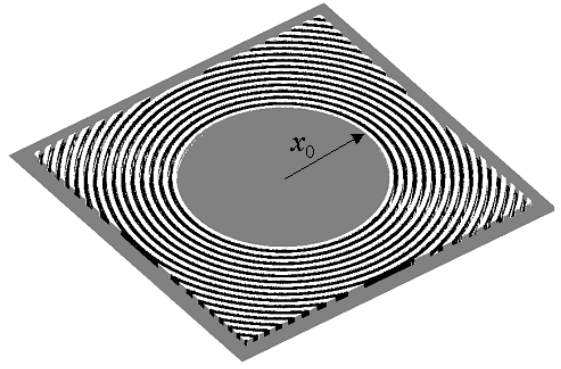

A

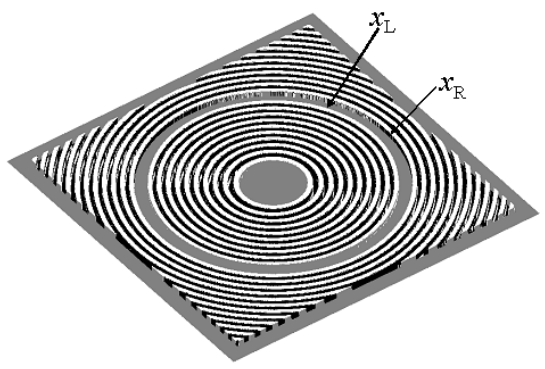

B

Fig. 1. An illustration of (a) a Bragg disk resonator and (b) an annular defect mode (ring) resonator structure.

We assume that the dielectric coefficient $\varepsilon(\rho)$ is given by $\varepsilon=n_{0}^{2}$ outside the grating region and by $\varepsilon(\rho)=n_{0}^{2}+\Delta \varepsilon(\rho)$ within the grating. Substituting the dielectric coefficient profile and introducing the normalized radius $x=k_{0} n_{0} \rho$ yield the wave equations

$$
\left\{\begin{array}{l}
x^{2} E_{x x}+x E_{x}+\left[x^{2}\left(1+\frac{\Delta \varepsilon(x)}{n_{0}^{2}}\right)-m^{2}\right] E=0, \\
x^{2} E_{x x}+x E_{x}+\left[x^{2}-m^{2}\right] E=0,
\end{array}\right.
$$

The electrical field in the unperturbed region is given by a superposition of the Hankel functions of the first and the second kind

$$
E(x)=\tilde{A} \cdot H_{m}^{(1)}(x)+\tilde{B} \cdot H_{m}^{(2)}(x) .
$$

The Hankel functions are the radial counterparts of the complex exponents in the Cartesian system in the sense that they correspond to radially incoming and outgoing waves $-H_{m}^{(1,2)}(x) \Leftrightarrow$ $\exp ( \pm i x)$. When the perturbation $\Delta \varepsilon(x)$ is small compared to $n_{0}^{2}$, the solution to (4a) describing the field in the grating region can be written as

$$
E(x)=A(x) \cdot H_{m}^{(1)}(x)+B(x) \cdot H_{m}^{(2)}(x)
$$

where the amplitudes $A(x)$ and $B(x)$ are assumed to vary slowly (as a function of $x$ ) compared to the Hankel functions, i.e., they are the slowly varying envelope approximation.

We start by solving for the field in the perturbed region. Introducing (6) into (4a) and neglecting the second derivatives of $A(x)$ and $B(x)$ yields [using (4b)]

$$
\begin{array}{r}
\frac{d A}{d x} \cdot\left(2 \frac{d H_{m}^{(1)}}{d x}+\frac{H_{m}^{(1)}}{x}\right)+\frac{d B}{d x} \cdot\left(2 \frac{d H_{m}^{(2)}}{d x}+\frac{H_{m}^{(2)}}{x}\right) \\
+\frac{\Delta \varepsilon(x)}{n_{0}^{2}}\left(A \cdot H_{m}^{(1)}+B \cdot H_{m}^{(2)}\right)=0 .
\end{array}
$$

Unlike Cartesian coupled mode theory (CMT) [17], (7) is quite complicated and it is not obvious what perturbation $\Delta \varepsilon(x)$ is required in order to couple power between the incoming and outgoing fields. In previous studies [6]-[8], the asymptotic expansion of the Hankel functions for large $x$ was used, replacing the Hankel functions with complex exponentials. Under this assumption, the required perturbation profile becomes a periodic function of $x$, which is similar to the Cartesian case. Here, we follow a similar route, but instead of introducing the conven- tional asymptotic expansion we employ the following approximation for large $x$ (see Appendix A for more details):

$$
\begin{aligned}
& \frac{H_{m}^{(1,2)}}{x}<<\frac{d H_{m}^{(1,2)}}{d x} \\
& \frac{d H_{m}^{(1,2)}}{d x} \approx \pm i \cdot H_{m}^{(1,2)} .
\end{aligned}
$$

Apparently, (8) is an excellent approximation (better than the conventional asymptotic expansion) for $x$ larger than the first zero of the Bessel function of order $m$ (especially for large $m$ ). The validity of this approximation is discussed further in Section III. Under these approximations, (7) can be written as

$$
\begin{aligned}
& 2 i H_{m}^{(1)}(x) \cdot A_{x}(x)-2 i H_{m}^{(2)}(x) \cdot B_{x}(x) \\
& \quad+\frac{\Delta \varepsilon(x)}{n_{0}^{2}}\left(A(x) \cdot H_{m}^{(1)}(x)+B(x) \cdot H_{m}^{(2)}(x)\right)=0 .
\end{aligned}
$$

Identifying $H_{m}^{(1)}$ and $H_{m}^{(2)}$ as the incoming and outgoing waves, it is clear that, in order for $\Delta \varepsilon$ to transfer power efficiently between them, the term $\Delta \varepsilon(x) H_{m}^{1}(x)$ should be proportional to $H_{m}^{2}(x)$ and, similarly, $\Delta \varepsilon(x) H_{m}^{2}(x)$ should be proportional to $H_{m}^{1}(x)$. For this to occur, $\Delta \varepsilon$ should have the following form:

$$
\Delta \varepsilon=\alpha \frac{H_{m}^{(2)}(x)}{H_{m}^{(1)}(x)}+\alpha^{*} \frac{H_{m}^{(1)}(x)}{H_{m}^{(2)}(x)} .
$$

Introducing (10) into (9) and keeping, as in the derivation of the Cartesian CMT, only the incoming and outgoing terms lead to coupled equations for $A$ and $B$. Since $H_{m}^{(1)}$ and $H_{m}^{(2)}$ are complex conjugates of each other, the ratio between them is twice the phase of the numerator and (10) can be rewritten as

$$
\begin{aligned}
\Delta \varepsilon(x)=\alpha \cdot \exp [ & \left.-2 i \cdot \operatorname{phase}\left(H_{m}^{(1)}(x)\right)\right] \\
& +\alpha^{*} \cdot \exp \left[2 i \cdot \operatorname{phase}\left(H_{m}^{(1)}(x)\right)\right] .
\end{aligned}
$$

It is interesting to note that the perturbation profile (11) exhibits fundamental resemblance to the required perturbation in the Cartesian system $\exp \left(2 i \beta_{s} z\right)$, where $\beta_{s}$ is the propagation factor of the wave that the perturbation is designed to reflect [17]. Since $\beta_{s} z=$ Phase $\left[\exp \left(i \beta_{s} z\right)\right]$, the perturbation profile in a Cartesian system can be written as

$\Delta \varepsilon_{\text {Cartesian }}=\exp \left( \pm 2 i \beta_{s} z\right)=\exp \left\{ \pm 2 i \cdot\right.$ Phase $\left.\left[\exp \left(i \beta_{s} z\right)\right]\right\}$.

The functional form of (12) is identical to the form of (11). Whether in Cartesian or cylindrical geometry, the generating functions are the eigen-modes of the wave equation in the ap- 
propriate coordinate system (plane waves for the Cartesian case and Hankel functions for the cylindrical case).

Equations (10) and (11) describe the perturbation profile which is required in order to couple between the incoming and outgoing waves for a specific wavelength $\lambda$ and azimuthal number $m$. In order to account for additional phenomena such as the wavelength dependence of the resonator mode, we introduce the following perturbation profile:

$$
\begin{aligned}
\Delta \varepsilon=\alpha \cdot \exp & {\left[-2 i \cdot \operatorname{phase}\left(H_{m}^{(1)}(x+\delta \cdot x)\right)\right] } \\
+ & \alpha^{*} \cdot \exp \left[2 i \cdot \operatorname{phase}\left(H_{m}^{(1)}(x+\delta \cdot x)\right)\right]
\end{aligned}
$$

where $\delta<<1$ represents a small deviation from the exact perturbation profile which is required for efficient coupling between the waves. $\delta$ can represent, for example, a small mismatch between the wavelength of the field which actually propagates in the resonator and the wavelength for which the radial reflector is designed. In this case, $\delta=\left(k_{\text {design }}-k_{0}\right) / k_{0}$.

Each Hankel function in (13) can be expanded by a Taylor series around $x$ as

$H_{m}(x+\delta \cdot x)=H_{m}(x)+H_{m}^{\prime}(x) \cdot \delta \cdot x+\cdots+\frac{1}{n !} H_{m}^{n}(x) \cdot(\delta \cdot x)^{n}$

where $H_{m}^{n}(x)$ represents the $n^{\text {th }}$ derivative of the Hankel function. Introducing the approximation for the Hankel derivative (8) into (14) yields

$$
\begin{aligned}
H_{m}^{(1)}(x+\delta \cdot x) & \approx H_{m}^{(1)}(x) \cdot \sum_{n=0}^{\infty} \frac{(i \delta \cdot x)^{n}}{n !} \\
& =H_{m}^{(1)}(x) \cdot \exp (i \delta \cdot x) .
\end{aligned}
$$

Introducing (15) into (13) yields the following perturbation profile:

$\Delta \varepsilon=\alpha \cdot \exp \left[-2 i \cdot \operatorname{phase}\left(H_{m}^{(1)}(x)\right)\right] \cdot \exp (-2 i \delta \cdot x)+c . c .$.

Substituting (16) into (9) and separating between the incoming and outgoing waves lead to the following set of coupled-waves equations for the amplitudes of the Hankel functions in the grating region (note that these equations are almost identical to the coupled waves equation in the Cartesian geometry):

$$
\begin{aligned}
2 i \cdot \frac{d A}{d x}+\frac{\alpha^{*}}{n_{0}^{2}} \cdot \exp (2 i \delta \cdot x) B & =0 \\
-2 i \cdot \frac{d B}{d x}+\frac{\alpha}{n_{0}^{2}} \cdot \exp (-2 i \delta \cdot x) A & =0 .
\end{aligned}
$$

The solution of (17) is given by

$$
\begin{aligned}
A(x)= & \left\{A_{1} \exp \left(\sqrt{k^{2}-\delta^{2}} \cdot x\right)\right. \\
& \left.+A_{2} \exp \left(-\sqrt{k^{2}-\delta^{2}} \cdot x\right)\right\} \cdot \exp (i \delta \cdot x) \\
B(x)= & -i \frac{\exp (-i \delta \cdot x)}{k^{*}} \\
& \cdot\left\{A_{1} \cdot\left(\sqrt{k^{2}-\delta^{2}}+i \delta\right) \cdot \exp \left(\sqrt{k^{2}-\delta^{2}} \cdot x\right)\right. \\
& \left.+A_{2} \cdot\left(-\sqrt{k^{2}-\delta^{2}}+i \delta\right) \cdot \exp \left(-\sqrt{k^{2}-\delta^{2}} \cdot x\right)\right\}
\end{aligned}
$$

where $k=\alpha / 2 n_{0}^{2}$ is the coupling coefficient.

The analysis in this section is 2-D and, therefore, does not account for losses that are induced by scattering from imperfec- tions of the resonator surfaces. The method to introduce such losses into the theory depends on their magnitude. Low losses can be modeled simply by multiplying the modal field by an azimuthally decaying term which can be estimated according to the field intensity at the interfaces [19]. High losses would also affect the optimal resonator design and require using complex-order Hankel functions in (6)-(16), where the imaginary part of the order is determined by the loss coefficient.

\section{DESIGN AND ANALYSIS OF BRAGG DISK/RING (DEFECT) RESONATORS}

In this section, we utilize (11) and (18) to design and analyze a Bragg annular resonator. The design and modal field are then compared to numerical solutions based on a matrix formalism [9].

We start with designing a disk resonator-a disk surrounded by radial Bragg grating [see Fig. 1(a)]. Since we attempt to design a resonator, we need to know the target wavelength $(\lambda=$ $\left.2 \pi / k_{0}\right)$ and the azimuthal number $m$ of the modal field. To realize maximal radial confinement, $\delta=0$ is preferred. Since the functional form of the perturbation is known [see (16)], the only parameters left to be determined are $\alpha$ (or, to be more precise, the phase of $\alpha$ ) and the disk radius $x_{0}$ [see Fig. 1(a)].

For high radial confinement (and simplicity), we assume that the number of Bragg layers is large so that the exponentially increasing term in (18) vanishes, i.e., $A_{1}=0$. Under the abovementioned assumptions, the wave amplitudes (18) and the electric field in the grating region $\left(x>x_{0}\right)$ are given by

$$
\begin{aligned}
A(x)= & A_{2} \exp (-|k| \cdot x) \\
B(x)= & i A_{2} \exp \left(-i \varphi_{k}\right) \cdot \exp (-|k| \cdot x) \\
E(x)= & A(x) H_{m}^{(1)}(x)+B(x) H_{m}^{(2)}(x) \\
= & A_{2} \exp (-|k| \cdot x) \\
& \cdot\left\{H_{m}^{(1)}(x)+i \cdot \exp \left(-i \varphi_{k}\right) \cdot H_{m}^{(2)}(x)\right\}
\end{aligned}
$$

where $\varphi_{k}$ is the phase of the coupling coefficient defined by $k=|k| \cdot \exp \left(i \varphi_{k}\right)$. Inside the disk $\left(x \leq x_{0}\right)$, the modal field is given by (5). The boundary conditions (BCs) require that the field is finite at $x=0$. This condition can be satisfied only if $\tilde{A}=\tilde{B}$ [see (5)] which means that the field at $x \leq x_{0}$ is given by $J_{m}(x)$ where $J_{m}$ is the Bessel function of order $m$ (the amplitude of the field in one of the regions can be selected arbitrarily). At $x=x_{0}$, both the field and its derivative must be continuous (for TE polarization) giving rise to the following characteristic equation:

$$
\frac{J_{m}^{\prime}\left(x_{0}\right)}{J_{m}\left(x_{0}\right)}+|k|=\frac{H_{m}^{\prime(1)}\left(x_{0}\right)+i \cdot \exp \left(-i \varphi_{k}\right) H_{m}^{\prime(2)}\left(x_{0}\right)}{H_{m}^{(1)}\left(x_{0}\right)+i \cdot \exp \left(-i \varphi_{k}\right) H_{m}^{(2)}\left(x_{0}\right)}
$$

Equation (20) has a relatively simple solution for $\varphi_{k}=-\pi / 2$. In this case, (20) is satisfied only if $x_{0}$ is a zero of the Bessel function, i.e., $J_{m}\left(x_{0}\right)=0$ and as a result $A_{2}=\exp \left(|k| x_{0}\right) / 2$. The required perturbation and total field are therefore given by $\Delta \varepsilon(x)=-4 n_{0}^{2}|k| \cdot \sin \left[2 \cdot \operatorname{phase}\left(H_{m}^{(1)}(x)\right)\right], \quad$ for $x>x_{0}$

$E(x)=\left\{\begin{array}{ll}J_{m}(x) & x \leq x_{0} \\ J_{m}(x) \exp \left(-|k| \cdot\left(x-x_{0}\right)\right) & x>x_{0}\end{array}\right\}$. 
A
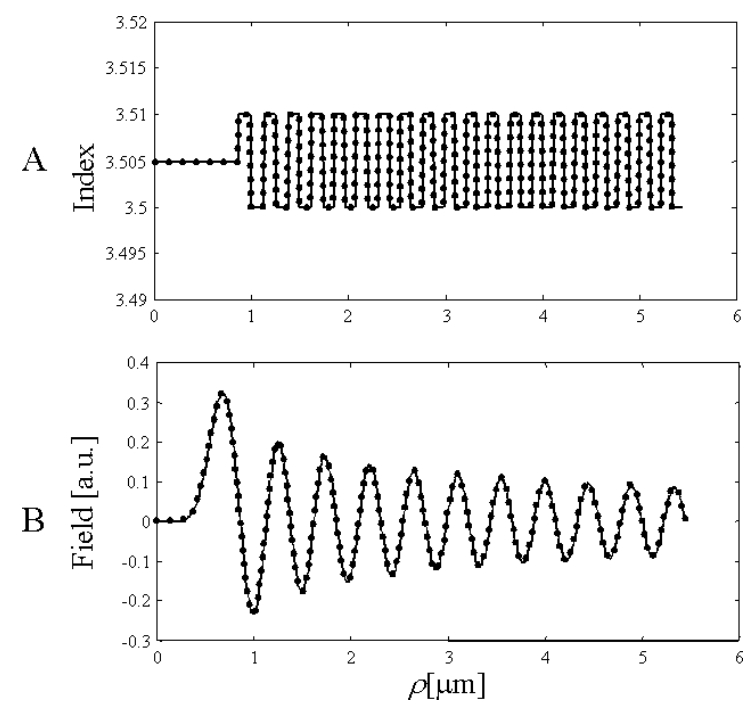

Fig. 2. Comparison between exact (solid) and approximated (dots) (a) layer structure and (b) radial mode profile of a Bragg disk resonator. $n_{0}=3.505$, $\Delta n_{0}=0.005, m=8$, and $\lambda=1.55 \mu \mathrm{m}$.

The fact that the required disk radius is a zero crossing of the Bessel function is very important since it means that the approximation (8) is valid throughout the whole perturbed region.

Equation (21) produces both the relevant perturbation term and the field profile. In order to radially confine the mode, the perturbation profile should include a term with the functional form of (21a). Consider, for example, the cylindric counterpart of the square wave corrugation commonly used in DBR lasers

$$
\begin{aligned}
\Delta \varepsilon(x)= & -\Delta \varepsilon_{0} \cdot \operatorname{sign}\left(\sin \left[2 \cdot \text { phase }\left(H_{m}^{(1)}(x)\right)\right]\right) \\
= & -\Delta \varepsilon_{0} \frac{4}{\pi} \cdot\left\{\sin \left[2 \cdot \text { phase }\left(H_{m}^{(1)}(x)\right)\right]\right. \\
& \left.+\frac{1}{3} \sin \left[2 \cdot 3 \cdot \text { phase }\left(H_{m}^{(1)}(x)\right)\right]+\cdots\right\}
\end{aligned}
$$

where $\Delta \varepsilon_{0}$ corresponds to the modulation depth of the index. The perturbation profile (22) has a square wave shape but is not periodic. The relevant term of the perturbation is the first coefficient in the Fourier expansion, yielding the following coupling coefficient:

$$
|k|=\frac{\Delta \varepsilon_{0}}{\pi \cdot n_{0}^{2}} .
$$

Fig. 2 depicts the first few micrometers of the index profile and the field profile for the analytical expressions (21) (dots) and the exact index and field profiles (solid line) calculated numerically using the transfer matrix method. The structure was designed for $n_{0}=3.505, \Delta n_{0}=0.005, m=8$, and $\lambda=1.55 \mu \mathrm{m}$. As shown in the figure, there is an excellent match between the analytical approximations and the exact numerical results, both for the field and the index profiles. Fig. 3 compares between the analytical and numerical field profiles for stronger perturbation: $n_{0}=3.5$ and $\Delta n_{0}=0.2$. As for the previous case, even though the perturbation is relatively strong (more than $5 \%$ ), there is an excellent match between the profiles.

We continue by employing the coupled-waves formalism to design a Bragg-based ring (defect) resonator [see Fig. 1(b)]. In this case, the modal field exhibits an exponential growing field in $0<x<x_{L}$ and exponentially decaying field in $x>x_{R}$

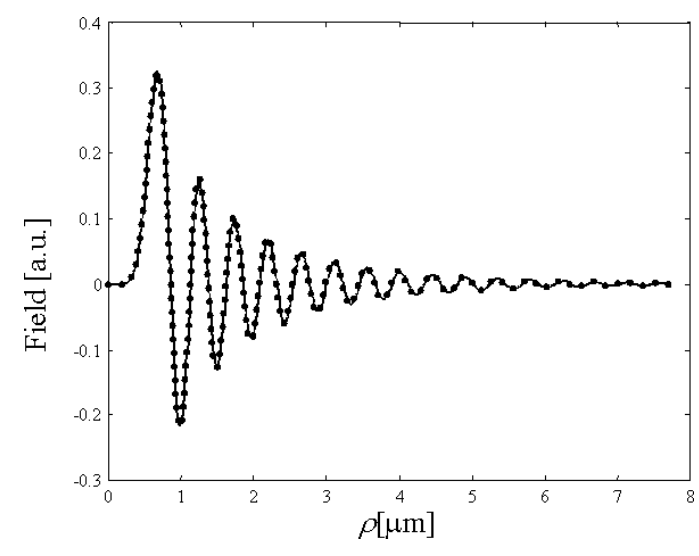

Fig. 3. Comparison between exact (solid) and approximated (dots) radial mode profile of a Bragg disk resonator utilizing higher index contrast. $n_{0}=3.5, \Delta n_{0}=0.2, m=8$, and $\lambda=1.55 \mu \mathrm{m}$.

[see Fig. 1(b)]. Practically, the field is guided by a radial defect located in the grating structure.

The modal electrical field in the structure is given by the following expression:

$E(x)=$

$\begin{cases}A_{1}(x) H_{m}^{(1)}(x)+B_{1}(x) H_{m}^{(2)}(x), & 0<x<x_{L} \text { Region I } \\ A_{2} \cdot H_{m}^{(1)}(x)+B_{2} \cdot H_{m}^{(2)}(x), & x_{L}<x<x_{R} \text { Region II } \\ A_{3}(x) H_{m}^{(1)}(x)+B_{3}(x) H_{m}^{(2)}(x), & x>x_{R} \text { Region III. }\end{cases}$

The boundary conditions are $A_{1}(0)=B_{1}(0)=1 / 2, E \rightarrow 0$ at $x \rightarrow \infty$, and that the field and its derivative must be continuous at $x_{L}$ and $x_{R}$. In the gratings regions (I and III), the slowly varying envelopes $A_{1,3}$ and $B_{1,3}$ are given in the general case by (18). For $\delta=0$ (maximal confinement) and assuming that the external reflector has a large number of Bragg layers, $A_{1,3}$ and $B_{1,3}$ are given by

$A_{1}(x)=A_{11} \exp (|k| x)+A_{12} \exp (-|k| x)$

$B_{1}(x)=-i \exp \left(i \varphi_{k 1}\right) \cdot\left[A_{11} \exp (|k| x)-A_{12} \exp (-|k| x)\right]$

$A_{3}(x)=A_{32} \exp (-|k| x)$

$B_{3}(x)=i \cdot \exp \left(i \varphi_{k 3}\right) \cdot A_{32} \exp (-|k| x)$

where $\varphi_{k 1,3}$ are the phases of the coupling factors in Regions I and III, respectively. As for the disk resonator, the amplitudes and the interface positions depend on the phase of the coupling coefficient. In order to find simple solutions, we choose $\varphi_{k}=$ $\pm \pi / 2$. It should be emphasized, however, that other options do exist. The BC at $x=0$ leads to $A_{11}=0$ for $\varphi_{k 1}=-\pi / 2$ and $A_{12}=0$ for $\varphi_{k 1}=\pi / 2$. Realization of an exponentially growing field in Region I requires $A_{11} \neq 0$ and therefore $\varphi_{k 1}=$ $\pi / 2$ and $E_{\mathrm{I}}(x)=J_{m}(x) \exp (|k| x)$.

In Region III, the field must decay and therefore the coupling coefficient at this region must be opposite to that of region I, i.e., $\varphi_{k 3}=-\pi / 2$, similar to the disk resonator design. The electric field in Region III is then given by $E_{\mathrm{III}}(x)=$ $A_{32} \cdot J_{m}(x) \exp (-|k| x)$. Since the field has only a real part at $x_{L}$ and $x_{R}$, it is clear that $A_{2}$ and $B_{2}$ in (24) must be complex conjugates of each other. The general modal solution is a superposition of the symmetric $\left(A_{2}=B_{2}\right)$ and the antisymmetric $\left(A_{2}=-B_{2}\right)$ solutions. For the symmetric case, the field 

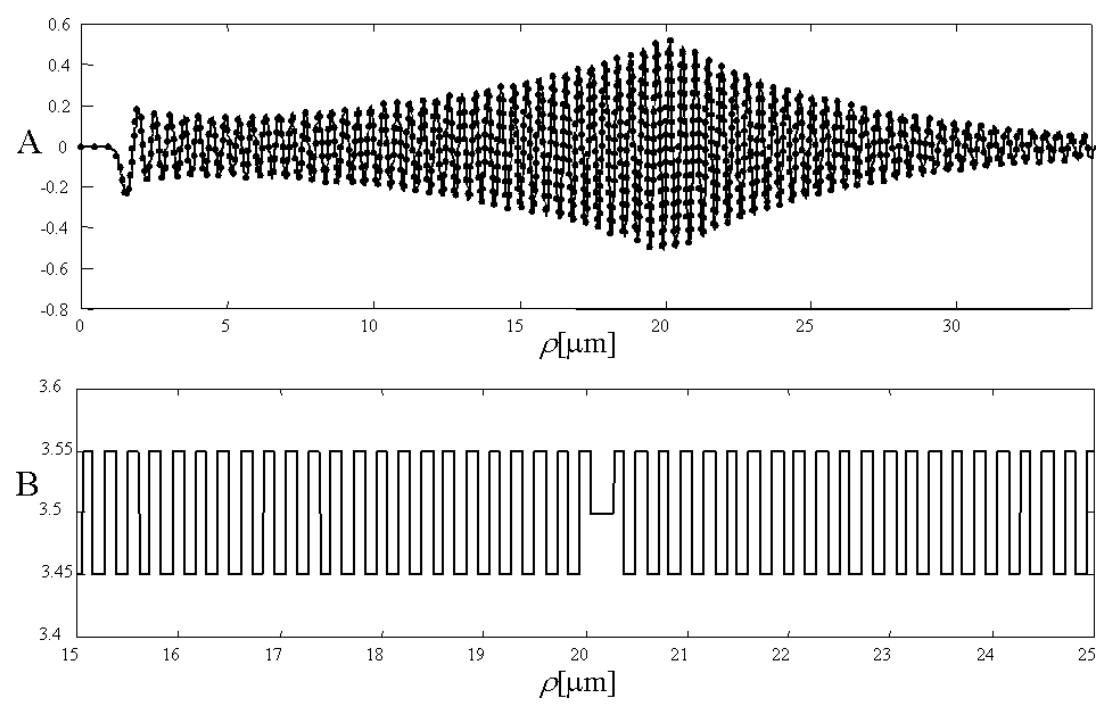

Fig. 4. (a) Comparison between exact (solid) and approximated (dots) radial mode profiles of an annular defect resonator. (b) Layer structure. $n_{0}=3.5, \Delta n_{0}=$ $0.05, m=20$, and $\lambda=1.55 \mu \mathrm{m}$.

in Region II is given by $E_{\mathrm{II}}=2 A_{2} \cdot J_{m}(x)$. Introducing the boundary conditions at $x_{L}$ and at $x_{R}$ yields the field profile and characteristic equations given by

$$
E(x)=\left\{\begin{array}{rr}
J_{m}(x) \exp (|k| x), \quad 0<x<x_{L} & \text { Region I } \\
J_{m}(x) \exp \left(|k| x_{L}\right), \quad x_{L}<x<x_{R} & \text { Region II } \\
J_{m}(x) \exp \left[-|k| \cdot\left(x-x_{L}-x_{R}\right)\right], & \\
x>x_{R} & \text { Region III }
\end{array}\right.
$$

$J_{m}\left(x_{L}\right)=0 ; \quad J_{m}\left(x_{R}\right)=0$

and the index perturbation profile is given by (27), shown at the bottom of the page. Fig. 4(a) depicts the field profile of the analytical (dots) and exact (solid line) field profiles for $n_{0}=$ 3.5, $\Delta n_{0}=0.05, m=20$, and $\lambda=1.55 \mu \mathrm{m}$. Fig. 4(b) depicts the required index profile for the same parameters. As for the disk resonator structure, there is an excellent match between the numerical and the analytical results.

\section{ANNUlar PHOtONIC BANDGAP RESONATORS}

In this section, we design and analyze resonators which are based on radial photonic crystal (PC) reflectors. Unlike the azimuthally symmetric Bragg resonators discussed in the previous section, it is difficult to employ a transfer matrix formalism for the analysis of PC resonators and, therefore, more complex numerical schemes (such as FDTD) are needed.

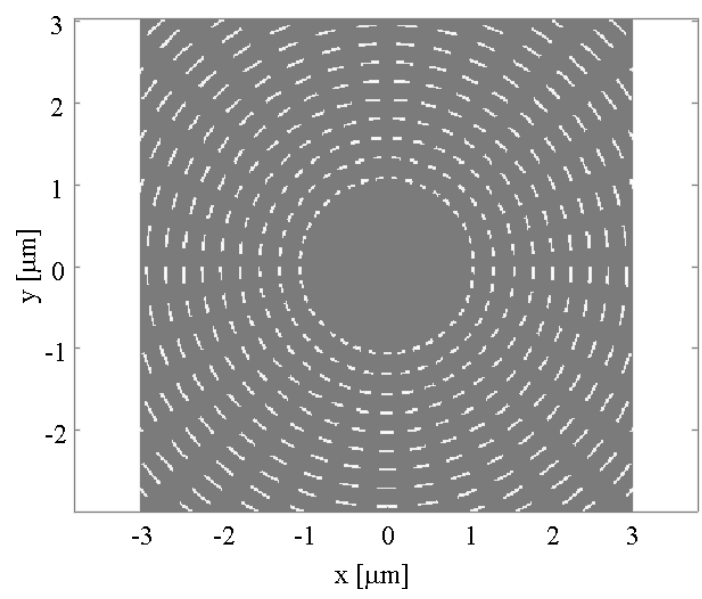

Fig. 5. The dielectric index profile of PC disk resonators. Black: material; white: air holes. The structure parameters are $n_{0}=3.5, m=8, \alpha_{1}=0.9$, $\alpha_{2}=0, l=37$, and $\lambda=1.55 \mu \mathrm{m}$.

To realize a PC reflector, the index profile should include a term of the form of (11). We suggest the following index profile:

$$
\begin{aligned}
& \varepsilon(\rho, \varphi)= n_{0}^{2}-\left(n_{0}^{2}-1\right) \\
& \cdot \Theta\left\{2 \cdot \operatorname{phase}\left(H_{m}^{(1)}(x)\right), \alpha_{1}\right\} \cdot \Theta\left[l \cdot \varphi, \alpha_{2}\right] \\
& \Theta(y, \alpha)= \begin{cases}1, & \sin (y) \geq \alpha \\
0, & \sin (y)<\alpha\end{cases}
\end{aligned}
$$

where $-1<\alpha_{1}, \alpha_{2}<1, n_{0}$ is the material index of refraction, and $l$ is the azimuthal number of the index profile. The structure

$$
\Delta \varepsilon(x)=\left\{\begin{array}{llr}
\Delta \varepsilon_{0} \cdot \operatorname{sign}\left\{\sin \left[2 \cdot \operatorname{phase}\left(H_{m}^{(1)}(x)\right)\right]\right\}, & 0<x<x_{L} & \text { Region I } \\
0, & x_{L}<x<x_{R} & \text { Region II } \\
-\Delta \varepsilon_{0} \cdot \operatorname{sign}\left\{\sin \left[2 \cdot \operatorname{phase}\left(H_{m}^{(1)}(x)\right)\right]\right\}, & x>x_{R} & \text { Region III }
\end{array}\right.
$$


consists a dielectric material with refractive index $n_{0}$ perforated by air holes. Fig. 5 depicts the dielectric index profile (28) for $n_{0}=3.5, m=8, \alpha_{1}=0.9, \alpha_{2}=0, l=37$, and $\lambda=1.55 \mu \mathrm{m}$. This index profile is the radial equivalent of a rectangular photonic bandgap crystal with rectangular holes. The perforation function $\Theta(y, \alpha)$ can be interpreted as a generalized rectifier of $\sin (y)$, generating square "pulses" only where $\sin (y)>\alpha$. The larger $a$ is, the smaller the "pulses" are. The index profile (28) includes two $\Theta$ functions- the first generates annular slits centered on the maxima of $\sin \left[2 \cdot\right.$ phase $\left.\left(H_{m}^{(1)}(x)\right)\right]$ with widths that are determined by $\alpha_{1}$. The second $\Theta$ function modulates these slits as a function of the angle $\varphi$ with angular frequency $l$ and a "duty cycle" which is determined by $\alpha_{2}$.

The Fourier expansion of the perforation function $\Theta(y, \alpha)$ is given by

$$
\begin{aligned}
\Theta(y, \alpha)= & \frac{\cos ^{-1}(a)}{\pi} \\
& -\sum_{m=1}^{\infty} \frac{(-1)^{m}-1}{m \pi} \cos \left[m \cdot \sin ^{-1}(a)\right] \cdot \sin (m y) \\
& -\sum_{m=1}^{\infty} \frac{(-1)^{m}+1}{m \pi} \sin \left[m \cdot \sin ^{-1}(a)\right] \cdot \cos (m y) .
\end{aligned}
$$

The term in the expansion of (28) which is responsible for the radial confinement of the modal field is $\Delta \varepsilon_{1,0}$ (i.e., first order in the radial expansion and zeroth order in the azimuthal expansion [20]) which is given by

$$
\Delta \varepsilon_{1,0}=-2 \cdot\left(n_{0}^{2}-1\right) \cos \left[\sin ^{-1}\left(\alpha_{1}\right)\right] \cdot \frac{\cos ^{-1}\left(\alpha_{2}\right)}{\pi^{2}} .
$$

It is important to note that $\Delta \varepsilon_{1,0}$ and, hence, the radial decay constant does not depend on the azimuthal number of the perturbation $l$. This is in contrast to the PC waveguide for which the transversal decay constant decreases for smaller lattice constants in the propagation direction [20]. This result is interesting, especially since it is obviously not true for every $l$ (consider for example $l=1$ or $l=2 \cdot m$ ). We expect this result to be correct only for $l$ 's which are larger than twice the azimuthal number of the modal field $-m$ (at $l=2 \cdot m$ there is strong coupling between the clockwise and counterclockwise propagating waves).

Once the coupling coefficient $k$ is known, the field profile is given by (26a) for a defect mode resonator or by (21b) for a disk resonator. The defect interfaces and disk radius are given by (26b) and (20), respectively. Unlike the Bragg resonators, the PC resonators cannot be modeled accurately by a transfer-matrix formalism and a different numerical scheme is required in order to verify the analytical results. Fig. 6 depicts an FDTD simulation of the structure shown in Fig. 5. A short pulse (1 ps) was injected into the structure and was left to propagate in the resonator until a steady-state solution was reached. As shown in the figure, the azimuthal number of the resonance field is indeed $m=8$, as it was designed to be. Fig. 7 depicts a comparison between the radial profile of the mode calculated by the FDTD simulation (dots) and the analytical solution calculated using the coupled mode formalism (solid line). As for the Bragg resonators, there is good agreement between the numerical results and the CMT prediction even though the index contrast in the

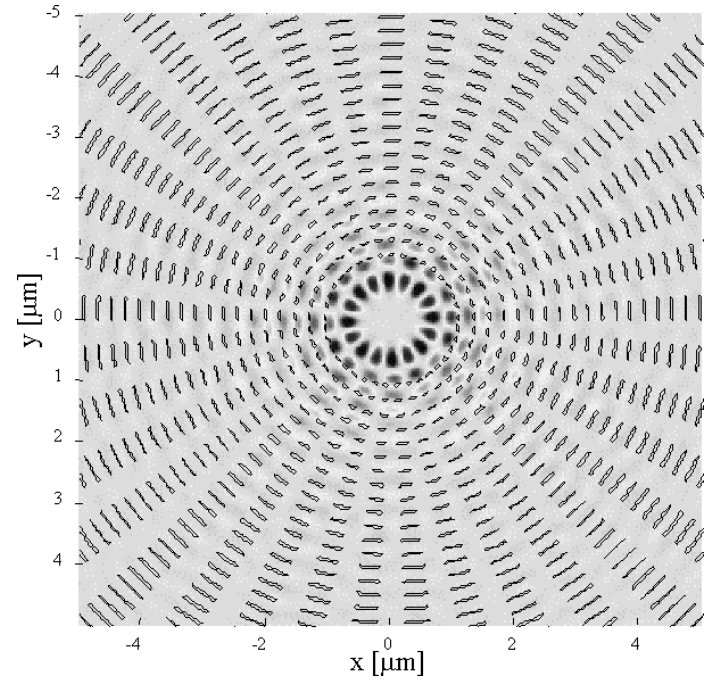

Fig. 6. The modal field of the structure depicted in Fig. 5, calculated by FDTD.

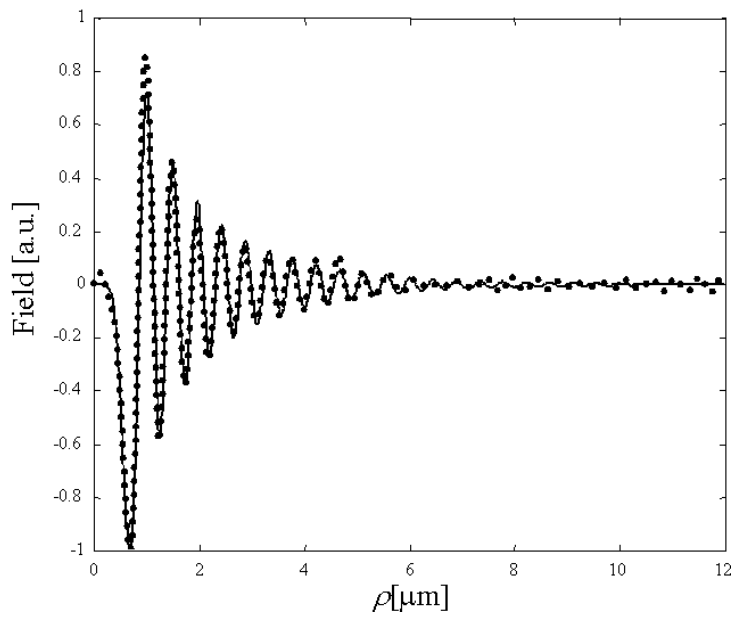

Fig. 7. Comparison between the radial profiles of the modal field of the structure depicted in Fig. 5, calculated by FDTD simulation (dots) and by the coupled waves formalism (solid line).

PC reflector region is fairly high. It is clear that the perturbative approach is valid only for $a_{1} \sim 1$ (i.e., small "filling fraction"). Comparing analytical predictions to the corresponding numerical solutions, we found that the analytical approach yields accurate results for $a_{1}>0.7$.

\section{SUMMARY AND CONCLUSION}

We have presented a novel perturbative approach to the analysis of annular resonators which are based on distributed reflectors. The derived coupled-waves equations were employed both for the design of the resonator as well as for the analysis of their modal field profiles. Excellent match was found between the approximated analytical results and the exact profiles calculated numerically by both the transfer matrix formalism and FDTD method.

For high reflectivity from the Bragg grating, the required radial dielectric coefficient perturbation term is the sine of twice the phase of the cylindrical incident wave phasor (Hankel function of order $m$ ). This result exhibits an interesting and fundamental resemblance to the Cartesian case for which the required 
perturbation can be determined in a similar manner, utilizing of course a plane wave instead of the Hankel function.

The good match between the analytical and numerical profiles of both the grating and the modal fields indicates that the perturbation approach presented here is a powerful and accurate tool for designing and analyzing annular Bragg resonators.

\section{APPENDIX}

\section{HANKEL FUNCTION DERIVATIVE APPROXIMATION}

The Hankel function of the first kind and its derivative can be represented by the following asymptotic expansions [21]:

$$
\begin{aligned}
H_{\nu}^{(1)}(x) \approx & \left(\frac{2}{\pi \cdot x}\right) \cdot \exp (i \varsigma) \sum_{s=0}^{\infty} i^{s} \frac{A_{s}(\nu)}{x^{s}} \\
H_{\nu}^{\prime(1)}(x) \approx & \left(\frac{2}{\pi \cdot x}\right) \\
& \cdot \exp (i \varsigma) \sum_{s=0}^{\infty} i^{s} \frac{A_{s}(\nu)}{x^{s}}\left(-\frac{1}{2 x}+i-\frac{s}{x}\right)
\end{aligned}
$$

where the expansion amplitudes and the common phase factor are given by

$$
\begin{aligned}
A_{s}(\nu) & =\frac{\left(4 \nu^{2}-1^{2}\right)\left(4 \nu^{2}-3^{2}\right) \cdots\left\lfloor 4 \nu^{2}-(2 s-1)^{2}\right\rfloor}{s ! \cdot 8^{s}} \\
\varsigma & =x-\frac{1}{2} \nu \pi-\frac{1}{4} \pi .
\end{aligned}
$$

It is clear that, if we keep only the leading term in (A2) when $x \rightarrow \infty$, the series (A2) is equal to (A1) multiplied by $i$, i.e.,

$$
H_{\nu}^{(1)}(x) \approx i \cdot H_{\nu}^{(1)}(x) .
$$

Since $H_{\nu}^{(2)}(x)$ is the complex conjugate of $H_{\nu}^{(1)}(x)$, it follows immediately that, in the limit of large $x, H_{\nu}^{\prime(2)}(x) \approx-i$. $H_{\nu}^{(2)}(x)$. Equivalently, in the limit of large $x$, the Bessel functions of the first and second kind admit the following relations, similar to the sine and cosine functions:

$$
\begin{aligned}
& J_{\nu}^{\prime}(x) \approx-Y_{\nu}(x) \\
& Y_{\nu}^{\prime}(x) \approx J_{\nu}(x) .
\end{aligned}
$$

Fig. 8 depicts the real (upper) and imaginary (lower) parts of the derivative of $H_{\nu}^{(1)}(x)$ (solid line), its approximation according to (A4) (dots), and an approximation which is based on the conventional asymptotic expansion of the Hankel function $-H_{\nu}^{(1)}(x)=\sqrt{2 / \pi x} \cdot \exp (i \varsigma)$ (dashed line) [22] for $\nu=8$. It is evident that the exact derivative and approximation (A4) coincide almost perfectly for values of $x$ which are larger than the first zero of the corresponding Bessel function (incidentally, the imaginary part of the dotted line). Although the standard approximation predicts correctly the amplitude of the Hankel derivative, it introduces a phase shift which is expressed by the position shift between the zeros of this approximation and of the exact function. This shift is especially important because, as we saw in Section II, the perturbation (and therefore the interfaces position in the case of Bragg reflector) is determined by the phase of the Hankel function. Therefore, designing a perturbation profile based on the standard asymptotic approximation would lead to inaccurate positioning of the gratings. The immediate consequence is
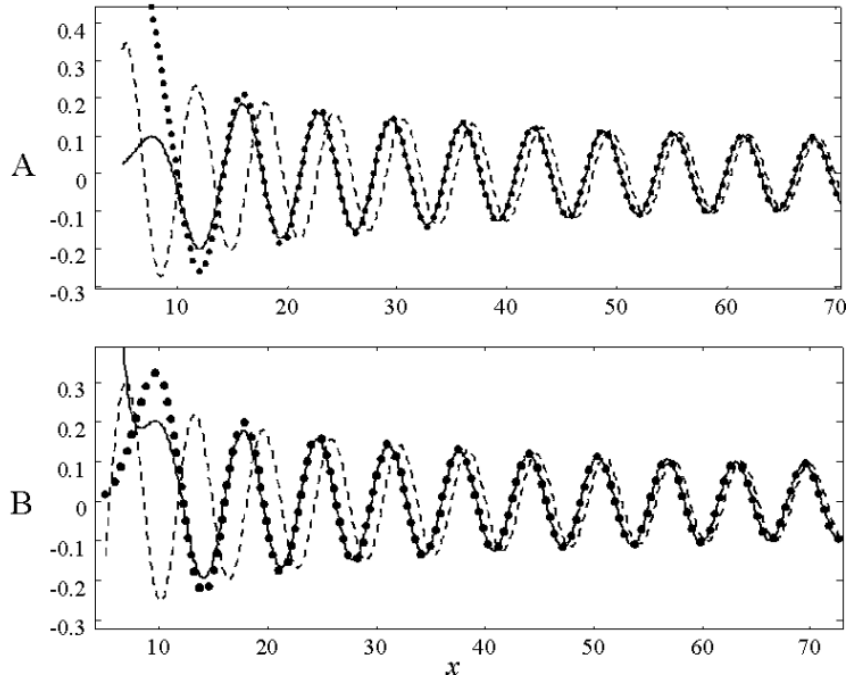

Fig. 8. Comparison between the exact derivative of $H_{8}^{(1)}(x)$ (solid line), its approximation according to (A4) (dots), and the derivative of the standard asymptotic approximation (dashed line). (a) Real part. (b) Imaginary part.

that the first Bragg layers do not contribute or even interfere with the radial confinement of the field, making it difficult to realize small radii Bragg resonators. The approximation (A4), however, does not introduce any phase shifts and therefore predicts more accurately the optimal positions of the interfaces.

\section{ACKNOWLEDGMENT}

The authors would like to thank S. Mookherjea and G. T. Paloczi for helpful discussions and comments.

\section{REFERENCES}

[1] A. Yariv, "Critical coupling and its control in optical waveguide-ring resonator systems," IEEE Photon. Technol. Lett., vol. 14, pp. 483-485, 2002.

[2] B. E. Little, "Ultracompact $\mathrm{Si}-\mathrm{SiO} 2$ microring resonator optical dropping filter," Opt. Lett., vol. 23, pp. 1570-1572, 1998.

[3] C. K. Madsen and J. H. Zhao, Optical Filter Design and Analysis: A Signal Processing Approach. New York: Wiley-Interscience, 1999.

[4] M. Toda, "Single-Mode behavior of a circular grating for potential diskshaped DFB lasers," IEEE J. Quantum Electron., vol. 26, pp. 473-481, 1990.

[5] X. H. Zheng and S. Lacroix, "Mode coupling in circular-cylindrical system and its application to fingerprint resonators," J. Lightwave Technol., vol. 8, pp. 1509-1516, 1990.

[6] T. Erdogan and D. G. Hall, "Circularly symmetric distributed feedback laser: An analysis," J. Appl. Phys., vol. 68, pp. 1435-1444, 1990.

[7] — , "Circularly symmetric distributed feedback laser: Coupled mode treatment of TE vector fields," IEEE J. Quantum Electron., vol. 28, pp. 612-623, 1992.

[8] X. M. Gong, A. K. Chen, and H. F. Taylor, "Lateral mode discrimination in surface emitting DBR lasers with cylindrical symmetry," IEEE J. Quantum Electron., vol. 30, pp. 1212-1218, 1994.

[9] M. A. Kaliteevski, R. A. Abram, V. V. Nikolaev, and G. S. Sokolovski, "Bragg reflectors for cylindrical waves," J. Mod. Opt., vol. 46, pp. 875-890, 1999.

[10] C. Wu, M. Svilans, M. Fallahi, T. Makino, J. Glinski, C. Maritan, and C. Blaauw, "Optically pumped surface-emitting DFB GaInAsP/InP lasers with circular grating," Electron. Lett., vol. 27, pp. 1819-1821, 1991.

[11] D. Labilloy, H. Benisty, C. Weisbuch, T. F. Krauss, C. J. M. Smith, R. Hourdré, and U. Oesterle, "High-finesse disk microcavity based on a circular Bragg reflector," Appl. Phys. Lett., vol. 73, pp. 1314-1316, 1998.

[12] A. Shaw, B. Roycroft, J. Hegarty, D. Labilloy, H. Benisty, C. Weisbuch, T. F. Krauss, C. J. M. Smith, R. Stanely, R. Hourdré, and U. Oesterle, "Lasing properties of disk microcavity based on circular Bragg reflector," Appl. Phys. Lett., vol. 75, pp. 3051-3053, 1999. 
[13] D. Ochoa, R. Hourdré, M. Ilegems, H. Benisty, T. F. Krauss, and C. J. M. Smith, "Diffraction of cylindrical Bragg reflectors surrounding an in-place semiconductor microcavity," Phys. Rev. B, vol. 61, pp. 4806-4812, 2000.

[14] S. Kim, H. Ryu, H. Park, G. Kim, Y. Choi, Y. Lee, and J. Kim, "Two-dimensional photonic crystal hexagonal waveguide ring laser," Appl. Phys. Lett., vol. 81, pp. 2499-2501, 2002.

[15] J. Scheuer and A. Yariv, "Two-Dimensional optical ring resonators based on radial Bragg resonance," Opt. Lett., vol. 28, pp. 1528-1530, 2003.

[16] — , "Annular Bragg defect mode resonators," J. Opt. Soc. Amer. B, vol. 20, pp. 2285-2291, 2003.

[17] A. Yariv, Optical Electronics in Modern Communications, 5th ed. New York: Oxford Univ. Press, 1997

[18] P. Yeh, A. Yariv, and E. Marom, "Theory of Bragg fiber," J. Opt. Soc. Amer., vol. 68, pp. 1196-1201, 1978.

[19] B. E. Little and S. T. Chu, "Estimating surface-roughness loss and output coupling in microdisk resonators," Opt. Lett., vol. 21, pp. 1390-1392, 1996.

[20] A. Yariv, "Coupled-wave formalism for optical waveguiding by transverse Bragg reflection," Opt. Lett., vol. 27, pp. 936-938, 2002.

[21] F. W. J. Olver, Asymptotics and Special Functions. New York: Academic, 1974

[22] G. N. Watson, Theory of Bessel Functions, 2nd ed. London, U.K.: Cambridge Univ. Press, 1952.

Jacob Scheuer (S'99-M'01) received the B.Sc. degrees in electrical engineering and physics with highest honors (summa cum laude) and the Ph.D. degree in electrical engineering from The Technion-Israel Institute of Technology, Haifa, Israel, in 1993 and 2001, respectively. His doctoral research involved theoretical and experimental study of the formation of complex light patterns and optical vortices in vertical-cavity surface-emitting lasers and the interactions between scalar/vector spatial solitons.

Currently, he is a Postdoctoral Associate in the Department of Applied Physics, California Institute of Technology, Pasadena. His current research interests include annular Bragg and photonic crystal resonator as well as coupled resonators optical waveguides (CROWs).
Amnon Yariv (S'56-M'59-F'70-LF'95) received the B.S., M.S., and Ph.D. degrees in electrical engineering from the University of California, Berkeley, in 1954, 1956, and 1958, respectively.

In 1959, he joined Bell Telephone Laboratories, Murray Hill, NJ, joining the early stages of the laser effort. In 1964, he joined the California Institute of Technology, Pasadena, as an Associate Professor of Electrical Engineering, becoming a Professor in 1966. In 1980, he became the Thomas G. Myers Professor of Electrical Engineering and Applied Physics. In 1996, he became the Martin and Eileen Summerfield Professor of Applied Physics and Professor of Electrical Engineering. On the technical and scientific sides, he took part (with various coworkers) in the discovery of a number of early solid-state laser systems, in the original formulation of the theory of nonlinear quantum optics; in proposing and explaining mode-locked ultrashort-pulse lasers, GaAs optoelectronics; in proposing and demonstrating semiconductor-based integrated optics technology; in pioneering the field of phase conjugate optics; and in proposing and demonstrating the semiconductor distributed feedback laser. His present research efforts are in the areas of nonlinear optics, semiconductor lasers, and WDM filters, especially the problem of monolithic integration of transistors, injection lasers, ultrafast $(<1 \mathrm{ps})$ semiconductor devices and phenomena, and the propagation of optical signals in fibers, including wavelength-division multiplexing (WDM). He has published widely in the laser and optics fields and has written a number of basic texts in quantum electronics, optics, and quantum mechanics. He is a founder and chairman-of-the-board of ORTEL Corporation (acquired by Lucent Technologies) and a founder and a board member of Arroyo Optics, Inc.

Dr. Yariv is a member of the American Physical Society, Phi Beta Kappa, the American Academy of Arts and Sciences, the National Academy of Engineering, and the National Academy of Sciences, and a Fellow of the Optical Society of America. He received the 1980 Quantum Electronics Award of the IEEE, the 1985 University of Pennsylvania Pender Award, the 1986 Optical Society of America Ives Medal, the 1992 Harvey Prize (shared with M. Gorbachev), and the 1998 Esther Beller Medal of the Optical Society of America. 